TRABAJOS ORIGINALES

\title{
Diversidad genética de papas nativas (Solanum spp.) del distrito de Vilcashuamán, Ayacucho- Perú, mediante AFLP
}

\section{Genetic diversity of native potatoes (Solanum spp.) from Vilcashuaman district, Ayacucho- Peru, using AFLP}

\section{Yessenia K. Remón Gamboa y Gilmar Peña Rojas*}

Laboratorio de Biología Celular y Molecular - Facultad de Ciencias Biológicas, Universidad Nacional de San Cristóbal de Huamanga, Ayacucho - Perú. *Autor para correspondencia

Email Gilmar Peña: gilmar_p@yahoo.com

Email Yessenia K. Remón: kyran920@gmail.com

\begin{abstract}
Resumen
En este presente trabajo, la diversidad genética de 30 morfotipos de papas nativas de Vilcashuamán (Ayacucho) fue evaluada mediante la técnica de polimorfismo de longitud de fragmentos amplificados (AFLP). La extracción de ADN se realizó con el método de CTAB modificado, usando hojas frescas de plantas de cuatro semanas de cultivo en invernadero. Partiendo de 200 mg de tejido vegetal se logró obtener entre 300 a $500 \mathrm{ng} / \mu \mathrm{L}$ de ADN de buena calidad. La digestión enzimática del ADN se realizó utilizando EcoRI y Msel, y se emplearon 12 combinaciones de primers, de las cuales se eligieron las dos combinaciones más polimórficas (E13 - M49 y E38 - M49). El análisis estadístico se realizó con el programa NTSYs 2.10 usando el coeficiente de Simple Matching logrando obtener valores de PIC (índice de contenido polimórfico) de 0.45 y 0.40 para las combinaciones E38 - M49 y E13 - M49, respectivamente. En total se lograron identificar 68 bandas claramente diferenciables, de las cuales el $55.8 \%$ fueron bandas polimórficas. El análisis de agrupamiento según el algoritmo UPGMA originó un dendograma con un índice de correlación cofenética de $r=0.7$; a un coeficiente de similitud de 0.6; se establecieron ocho grupos genéticos y a un coeficiente de 1 no se encontraron morfotipos duplicados. Los resultados obtenidos demuestran el alto poder informativo del AFLP y la alta variabilidad de las papas nativas estudiadas.
\end{abstract}

Palabras claves: Vilcashuamán; papas nativas; AFLP; diversidad genética; Solanum spp.

\section{Abstract}

In this article, using the Amplified Fragment Lenght Polymorphism (AFLP) technique, we evaluated the genetic diversity of 30 native potatoes morphotypes from Vilcashuaman, Ayacucho. DNA extraction was done with the modified CTAB method, using fresh leaves of greenhouse plants of two weeks age. From $200 \mathrm{mg}$ of plant tissue, it was posible to obtain between 300 and $500 \mathrm{ng} / \mathrm{\mu L}$ of good quality DNA. The enzymatic digestion of the DNA was carried out using EcoRI and Msel, and 12 combinations of primers were used, from which the two most polymorphic combinations were chosen (E13 - M49 and E38 - M49). The statistical analysis was done with the NTSYs 2.10 program using the Simple Matching coefficient, obtaining values of PIC (polymorphic content index) of 0.45 and 0.40 for the combinations E38 - M49 and E13 - M49, respectively. In total, 68 clearly differentiable bands were identified, of which $55.8 \%$ were polymorphic bands. The cluster analysis according to the UPGMA algorithm originated a dendrogram with a cofenetic correlation index of $r=0.7$; at a coefficient of similarity of 0.6 , eight genetic groups were established and at a coefficient of 1 , no duplicate morphotypes were found. The results obtained show the high informative power of the AFLP and the high variability of the native potatoes studied.

Keywords: Vilcashuaman; native potatoes; AFLP; genetic diversity; Solanum spp.

Citación:

Remón Gamboa Y.K. \& G. Peña Rojas. 2018. Diversidad genética de papas nativas (Solanum spp.) del distrito de Vilcashuamán, AyacuchoPerú, mediante AFLP. Revista peruana de biología 25(3): 259 - 266 (Agosto 2018). doi: http://dx.doi.org/10.15381/rpb.v25i3.15209

Fuentes de financiamiento: PROCYT N²33-2008-CONCYTEC-OAJ.

Presentado: $\quad$ 09/08/2017

Aceptado: $\quad 22 / 07 / 2018$

Publicado online: $25 / 09 / 2018$
Información sobre los autores:

YKRG, Coleccion y analisis de la diversidad de papas nativas medianteAFLP GPR analisis de la diversidad de !as papas nativas mediante AFLP. Ambos redactaron, revisaron y aprobaron el manuscrito.

Los autores no incurren en conflictos de intereses. 


\section{Introducción}

Las papas nativas y silvestres son los ancestros de todas las variedades modernas de papa que existen en el mundo (Ochoa 1999). Entre 6000 y 10000 ańos atrás en los Andes, las primeras papas cultivadas fueron seleccionadas (Spooner et al. 2005) por generaciones de agricultores que produjeron una gran cantidad de variantes cultivadas a partir de papas silvestres (Ochoa 1999, Huamán \& Spooner 2002). En la actualidad, esas variedades se encuentran distribuidas en toda América, desde el sur de los Estados Unidos hasta la isla de Chile, siendo el Perú la que posee la mayor diversidad (Hijmans et al. 2002).

Spooner et al. (2005) establecieron como el centro de origen de la papa la región norte del lago Titicaca, al sur de Perú, donde su cultivo estuvo ligada al desarrollo de la cultura Tiahuanaco. Posteriormente, la segunda gran expansión del cultivo de la papa, se dio durante la formación del imperio Inca; aunque, el cultivo de la papa ya estaba arraigado en las regiones andinas (Hawkes 1990, Ochoa 1999). Por esta razón, la mayor diversidad genética conservada por los agricultores se encuentra en algunas regiones de la sierra como Ayacucho (Spooner et al. 2005, Hawkes 1990).

Algunos estudios señalan que la domesticación de la papa tuvo lugar en la sierra del Perú, probablemente en la región que incluye los actuales departamentos de Huánuco, Cerro de Pasco, Junín, Huancavelica, Apurímac, Ayacucho, Cusco y Puno (Hawkes 1990, Morales 2007), y donde los pobladores andinos, en la actualidad basan su seguridad alimentaria en la papa y junto a su conocimiento ancestral constituyen la razón de encontrar hasta la fecha las mismas formas y colores que conocieron los incas y sus predecesores, cumpliendo así un rol decisivo en la conservación de las variedades (Hawkes 1990, Ochoa 1999, Morales 2007, Salvatierra 2013). Sin embargo, la diversidad de las papas nativas se está perdiendo debido a la presencia de variedades mejoradas, el uso excesivo de agroquímicos y el cambio climático; motivo por el cual, el estudio y la caracterización genética de la diversidad de papas nativas es imprescindible para el establecimiento, mantenimiento y conservación en los bancos de germoplasma (Huamán 1986, Túpac 2001, Gutiérrez 2008, Salvatierra 2013).

La caracterización morfológica de los tubérculos puede considerarse como fuente de información para identificar genotipos en los bancos de germoplasma (Calliope et al. 2018). Madrońero et al. (2013) señalan que las mejores variables para la caracterización son aquellas relacionadas con el color de tallo, color de brote y color y forma de baya. No obstante, muchos de ellos pueden ser alterados por enfermedades, variar con las condiciones ambientales; más aún, estas evaluaciones son subjetivas y están condicionadas al criterio y experiencia de cada investigador (Leitch et al. 1998, Coulibaly et al. 2003); por tanto, la caracterización morfológica debe ser complementada con estudios de caracterización por marcadores moleculares.

Los marcadores moleculares permiten la detección de cambios moleculares en el genoma, generan datos altamente confiables y replicables (Albertini et al. 2003), obteniéndose un "perfil molecular" característico para cada variedad, independiente de las condiciones ambientales y del crecimiento de la planta (Morrell et al. 1995, Moscoe \& Emshwiller 2015). Tanto los AFLPs (Amplified Fragment Lenght Polymorphisms, Vos et al., 1995) y los SSR (microsatélites, Chambers \& MacAvoy, 2000) tienen sus ventajas y desventajas y, su aplicación dependerá fundamentalmente del tipo de cultivo, disponibilidad de recursos y la capacitación del personal (Montalvo et al. 2012, Canales et al. 2003, Guichoux et al. 2011).

Los marcadores AFLP han sido ampliamente empleados porque permiten una exploración rápida de los polimorfismos del genoma, generan un gran número de bandas por experimento, son altamente reproducibles, no necesita información previa del genoma para su aplicación, tiene carácter codominante y permite el uso de pequeńas cantidades de ADN (Vos et al. 1995, Montalvo et al. 2012). El procedimiento AFLP consiste en la digestión del genoma con enzimas de restricción y amplificación selectiva de fragmentos resultantes. Estos fragmentos son posteriormente evaluados como presente o ausente en los resultados de electroforesis (Moscoe \& Emshwiller 2015). Wang et al. (2017) afirman que el AFLP puede producir patrones más informativos y es ampliamente utilizado para evaluar la diversidad genética de germoplasmas de papa, y en definitiva Narváez et al. (2000) y Nunziata et al. (2010) sugieren su uso en la caracterización de la diversidad. Es así que, existen numerosos trabajos de investigación sobre la diversidad y caracterización genética de papas usando dicho marcador molecular (Solano et al. 2007, Esfahani et al. 2009, Yildirim et al. 2010, Wang et al. 2011, Gonzales \& Peña 2014, Sánchez 2017, Wang et al. 2017). En el presente trabajo se utilizó el AFLP para caracterizar la diversidad genética de 30 morfotipos de papas nativas de Vilcashuamán que hasta la fecha no existe ningún antecedente sobre estudios de esta naturaleza.

\section{Material y métodos}

Colecta de las muestras.- Se colectaron 30 morfotipos de tubérculos (Tabla 3 ) de la provincia de Vilcashuamán (1340'43.3"S-1340'41.72"S y 7352'40.92"W$73^{\circ} 52^{\prime} 35.48^{\prime \prime} \mathrm{W}$, entre 3731 a $3760 \mathrm{~m}$ de altitud). Los tubérculos fueron sembrados en el vivero de la Facultad de Ciencias Biológicas de la Universidad Nacional de San Cristóbal de Huamanga, a una temperatura promedio de $17{ }^{\circ} \mathrm{C}$, utilizando bolsas de polietileno $15 \times 20 \mathrm{~cm}$ que contenía tierra, arena y musgo en una proporción 3:2:1 respectivamente. Las hojas jóvenes de cuatro semanas de cultivo, fueron colectadas en placas de petri y posteriormente se congelaron a $-20^{\circ} \mathrm{C}$ por $24 \mathrm{~h}$ para el proceso de extracción del ADN.

Extracción, determinación y concentración de ADN.- La extracción de $\mathrm{ADN}$ se realizó por duplicado con el método CTAB (CIP 1997) modificado, a partir de $200 \mathrm{mg}$ de tejido fresco. Se agregó $1 \mu \mathrm{L}$ de RNAsa por cada $100 \mu \mathrm{L}$ de muestra de ADN, posteriormente fueron diluidas en agua Milli Q libre de nucleasas a una concentración de $50 \mathrm{ng} / \mu \mathrm{L}$. Se evaluó la calidad y concentración del ADN en gel de agarosa al 1\%, para lo cual se tomaron $2 \mu \mathrm{L}$ del ADN resuspendido y se diluyó en $10 \mu \mathrm{L}$ de tampón de carga Runsafe ${ }^{\circledast}$ para su observación en el transluminador UV; los resultados se corroboraron con una lectura en el NanoDrop ${ }^{\circledR} 2000$

Técnica de AFLP.- La técnica se realizó según el protocolo descrito por Vos et al. (1995), adaptado y modificado por el CIP (1998).

Digestión, se utilizó 500 ng de ADN genómico, agua libre de nucleasas, buffer de reacción 10X, albúmina de suero bovino y enzimas de restricción: 5 unidades (U) de MseI y 20U de 
Tabla 3. Caracterización morfológica de los tubérculos de 30 morfotipos de papas nativas procedentes de la provincia de Vilcashuamán, Ayacucho - 2015

\begin{tabular}{|c|c|c|c|c|c|c|c|}
\hline $\mathrm{N}^{\circ}$ & MORFOTIPO & $\begin{array}{c}\text { Color } \\
\text { predominante } \\
\text { de la piel }\end{array}$ & $\begin{array}{l}\text { Color } \\
\text { secundario de } \\
\text { la piel }\end{array}$ & $\begin{array}{l}\text { Color } \\
\text { predominante de } \\
\text { la pulpa }\end{array}$ & $\begin{array}{c}\text { Color } \\
\text { secundario } \\
\text { de la pulpa }\end{array}$ & $\begin{array}{l}\text { Forma general del } \\
\text { tubérculo }\end{array}$ & $\begin{array}{l}\text { Profundidad de } \\
\text { los ojos }\end{array}$ \\
\hline 1 & Chaolina & Amarillo & Ausente & Amarillo & Ausente & Oblongo (reniforme) & Superficial \\
\hline 2 & Rosa huayta & Rojo-morado & Ausente & Amarillo claro & Ausente & Redondo & Medio \\
\hline 3 & Qeqorani & Amarillo & Ausente & Crema & Morado & Oblongo & Superficial \\
\hline 4 & Renacimiento & Amarillo & Ausente & Crema & Ausente & Oblongo & Superficial \\
\hline 5 & wana Wayro & Negruzco & Marrón & Blanco & Ausente & $\begin{array}{l}\text { Oblongo-alargado } \\
\text { (concertinado) }\end{array}$ & Profundo \\
\hline 6 & Asnupa runtun & Negruzco & Marron & Blanco & Violeta & Oblongo & Superficial \\
\hline 7 & Tambina & Rojo-morado & Ausente & Crema & Morado & Oblongo & Profundo \\
\hline 8 & Duraznillo & Rojo-morado & Amarillo & Crema & Ausente & Redondo & Medio \\
\hline 9 & Turupa yawarnin & Rojo-morado & Marron & Rojo & Blanco & Elíptico (fusiforme) & Superficial \\
\hline 10 & Quello camotillo & Amarillo & Ausente & Crema & Ausente & Ovobado & Superficial \\
\hline 11 & Quello Wenqos & Amarillo & Ausente & Amarillo & Ausente & Alargado (falcado) & Sobresaliente \\
\hline 12 & Puka huayro & Rojo-morado & Ausente & Amarillo intenso & Ausente & $\begin{array}{l}\text { Oblongo alargado } \\
\text { (concertinado) }\end{array}$ & Profundo \\
\hline 13 & Caspas & Negruzco & Rosado & Amarillo intenso & Ausente & Oblongo (tuberosado) & Muy profundo \\
\hline 14 & Leona & Negruzco & Marron & Blanco & Violeta & Oblongo & Profundo \\
\hline 15 & Yawar huayqo & Rojo-morado & Amarillo & Rojo-morado & Amarillo & Oblongo & Profundo \\
\hline 16 & Peruanita & Rojo-morado & Amarillo & Amarillo & Ausente & Oblongo & Profundo \\
\hline 17 & Allqa toyros & Amarillo & Rosado & Crema & Ausente & $\begin{array}{l}\text { Oblongo alargado } \\
\text { (omprimido) }\end{array}$ & Medio \\
\hline 18 & Wachwapa qallon & Rosado & Blanco crema & Crema & Rosado & Oblongo & Superficial \\
\hline 19 & Allqa yuraq sisa & Amarillo & Negruzco & Violeta & Amarillo & Comprimido & Superficial \\
\hline 20 & $\begin{array}{l}\text { Puka Llumchuy } \\
\text { waqachi }\end{array}$ & Rojo-morado & Ausente & Blanco & rojo & Oblongo Tuberosado & Muy profundo \\
\hline 21 & Llamapa ñawin & negruzco & Ausente & Amarillo & Ausente & Comprimido & Profundo \\
\hline 22 & Yana camotillo & Negruzco & Rojo-morado & Crema & Ausente & Ovobado & Superficial \\
\hline 23 & $\begin{array}{l}\text { Allqa llumchuy } \\
\text { huaqachi }\end{array}$ & Negruzco & Amarillo & Crema & Ausente & Oblongo Tuberosado & Muy profundo \\
\hline 24 & Yuraq wenqos & Crema & Ausente & Crema & Ausente & Alargado (falcado) & Sobresaliente \\
\hline 25 & Yuraq sisa & Rosado & Ausente & Blanco & Ausente & Comprimido & Profundo \\
\hline 26 & Suytu camru & Amarillo & Rojo-morado & Blanco & Morado & Oblongo alargado & Medio \\
\hline 27 & Chontamoron & Negruzco & Amarillo & Blanco & Ausente & Ovobado & Superficial \\
\hline 28 & Roya puyru & Amarillo & Ausente & Crema & Ausente & Oblongo & Profundo \\
\hline 29 & Yana wenqos & Negruzco & Amarillo & Crema & Morado & Alargado (falcado) & Superficial \\
\hline 30 & Puka pumapa makin & Rojo-morado & Ausente & Amarillo & Rosado & Oblongo (digitado) & Medio \\
\hline
\end{tabular}

EcoRI. Posteriormente la reacción se incubó a $37^{\circ} \mathrm{C}$ toda la noche, luego a $65^{\circ} \mathrm{C}$ por 15 minutos. La obtención del ADN digerido fue verificado en gel de agarosa al $1 \%$, observándose un barrido de $\mathrm{ADN}$ característico.

Ligación, se tomó $20 \mu \mathrm{L}$ ADN digerido, $120 \mathrm{U}$ de $\mathrm{T}_{4} \mathrm{ADN}$ ligasa, $50 \mu \mathrm{M}$ de adaptador MseI, $10 \mu \mathrm{M}$ de adaptador EcoRI, buffer de reacción y agua destilada libre de nucleasas, posteriormente la solución se incubó a temperatura ambiente $\left(20^{\circ} \mathrm{C}\right)$ toda la noche y finalmente se realizó una dilución 1:5.
Reacción de pre-amplificación (00/00), se preparó el master mix conteniendo agua destilada libre de nucleasas, buffer 10X, dNTPs, $\mathrm{MgCl}_{2}$ y primers: $10 \mu \mathrm{M}$ de EcoRI y $10 \mu \mathrm{M}$ de MseI sin nucleótidos adicionales $(00 / 00)$; a la mezcla preparada se adicionó $5 \mu \mathrm{L}$ de $\mathrm{ADN}$ ligado/diluido. La reacción de preamplificación de PCR se realizó en el termociclador Applied Biosystems ${ }^{\oplus}$, el programa usado fue: 1 ciclo $\left(72{ }^{\circ} \mathrm{C} / 2 \mathrm{~min}\right), 24$ ciclos $\left(94^{\circ} \mathrm{C} / 30 \mathrm{seg}, 56^{\circ} \mathrm{C} / 60 \mathrm{seg}\right)$ y finalmente de $72{ }^{\circ} \mathrm{C}$ / 5 min, concluyendo la reacción a una temperatura de $4{ }^{\circ} \mathrm{C}$. 
Amplificación selectiva $(+3 /+3)$, se determinó 12 combinaciones de iniciadores (+ 3 nucleótidos selectivos): E13 - M49; E41 -M48; E35 - M49; E38 - M50; E35 - M37; E39 - M48; E39 - M49; E38 - M49; E12 - M32; E13 - M37; E37 - M50; E38 - M61. Se usó $2 \mu \mathrm{L}$ de ADN pre-amplificado/diluido (1:3), $5 \mathrm{mM}$ de dNTPs, $0.75 \mathrm{U}$ de Taq polimerasa, $100 \mathrm{ng}$ de MseI(+3) y $15 \mathrm{ng}$ de $\mathrm{EcoRI}(+3)$. El programa usado en el termociclador fue: 1 ciclo inicial $\left(94^{\circ} \mathrm{C} / 20 \mathrm{seg}, 65^{\circ} \mathrm{C} / 30 \mathrm{seg}\right.$ y $72{ }^{\circ} \mathrm{C} / 2$ min), seguido de 8 ciclos en el que la temperatura de hibridación se redujo en $1{ }^{\circ} \mathrm{C}$; a continuación, la temperatura se mantuvo constante a $56^{\circ} \mathrm{C}$ para los 20 ciclos restantes.

Electroforesis en geles de poliacrilamida y tinción.- Los productos de la amplificación selectiva se separaron en geles denaturantes de poliacrilamida al $6 \%$ y la visualización de dichos fragmentos se realizó con la tinción de nitrato de plata.

Escaneado y lectura de bandas amplificadas.- La lectura de las bandas se realizó utilizando los geles con mejor resolución y se enumeraron consecutivamente empezando desde el primer individuo del primer carril, su enumeración correspondió a 1 como presencia y 0 como ausencia para cada morfotipo respectivamente.

Análisis estadístico.- Los datos se procesaron en una matriz binaria Excel y la similitud genética se calculó utilizando el coeficiente de similitud Simple Matching, método del grupo par no ponderado con promedios aritméticos (UPGMA) usando el programa NTSYS 2.10

\section{Resultados y discusión}

Utilizando el método CTAB (CIP 1997) modificado se logró obtener ADN de buena calidad cuyas concentraciones oscilaron entre 300 a $500 \mathrm{ng} / \mu \mathrm{L}$ por $200 \mathrm{mg}$ de tejido vegetal, los resultados obtenidos coinciden con los trabajos descritos por Gonzáles \& Peña (2014) en la caracterización de las papas nativas de Chungui y, Sánchez (2017) en el estudio de la variabilidad genética en accesiones de papa. En ambos casos, obtuvieron concentraciones similares en el estudio de la diversidad de la papa tanto en Perú como en Colombia. No obstante, Kim et al. (1998) reportó un mejor rendimiento y una excelente calidad del ADN utilizando el método descrito por Dellaporta et al. (1983). Iglesias et al. (2003) al comparar tres técnicas de extracción, incluyendo las dos técnicas mencionadas anteriormente, sugieren también el método CTAB. Fierro (2004) señala que el método CTAB es una buena técnica para la extracción de $\mathrm{ADN}$ de plantas que tienen un alto contenido de polisacáridos y metabolitos secundarios.

Análisis de la diversidad genética por AFLP.- como se muestra en la Tabla 1, en el presente trabajo se obtuvo un total de 68 bandas, de las cuales el 55.9\% resultaron ser bandas polimórficas y un promedio de 34 bandas polimórficas por iniciador, los resultados obtenidos coinciden con Esfahani et al. (2009) y Gonzales \& Peña (2014). No obstante, el número de bandas obtenidas es menor respecto a los estudios de Solano et al. (2007). De otro lado, Wang et al. (2011) y Yildirim et al. (2010) obtuvieron bandas superiores a 43 por combinación de iniciadores. Según Vos et al. (1995) una de las ventajas del uso de AFLP es la generación de gran cantidad de polimorfismo, siendo entre 50 a 100 bandas o locus por iniciador. Al respecto Wang et al. (2011) mencionan que el análisis de polimorfismo no es muy fiable cuando el número de locus o bandas obtenidas es por debajo de 20 y la fiabilidad de la información es estable al superar 70 locus. En el presente trabajo se probaron 12 combinaciones de iniciadores que también fueron utilizados por Yildirim et al. (2010); Wang et al. (2011) y, Gonzales \& Peńa (2014). La combinación de iniciadores E39 - M49 usada por Wang et al. (2011) y la combinación E37 - M50 utilizado por González y Peña (2014) no resultaron ser polimórficas para el presente estudio. No obstante, la combinación E38 - M49 fueron menos polimórficas en relación a la combinación E13-M49 que presentó 22 bandas polimórficas, a diferencia de la combinación E38 - M49 que mostró sólo 16; por tanto, la combinación E13 - M49 resultó más polimórfica e informativa en el estudio de la diversidad de la papa de Vilcashuamán.

Asimismo, Moscoe et al. (2015) en el estudio de la diversidad de Oxalis tuberosa Molina, mencionan que los datos de AFLP revelaron 66 bandas con un promedio de 28.19 bandas presentes por muestra, valor superior que Wang et al. (2017), quienes usaron 10 combinaciones de iniciadores y obtuvieron 998 bandas en 288 colecciones de germoplasmas de papa, y de ellas 983 bandas fueron polimórficos con un promedio de 98.3 por combinación de iniciador. Wang et al. (2017) mencionan que la variación puede estar influenciada por el origen de las colecciones de papa, tipo de marcador y la metodología utilizada para el revelado de las bandas por electroforesis.

Contenido de Información polimórfica (PIC).- La combinación de iniciadores E13 - M49 mostraron 39 bandas, de las cuales el 58\% fueron polimórficas y con un valor de PIC 0.45, mayor que la combinación E38 - M49 con un PIC de 0.40 (Tabla 2), los valores obtenidos determinan la variabilidad de papas nativas en Vilcashuamán. Gonzales y Peña (2014) reportaron un resultado similar (0.43) para la caracterización molecular de pa-

Tabla 2. Valores del Índice de Contenido Polimórfico (PIC) para las combinaciones de iniciadores más polimórficas.

\begin{tabular}{cc}
\hline Combinación de iniciadores & $\begin{array}{c}\text { Índice de contenido polimórfico } \\
\text { (PIC) }\end{array}$ \\
\hline E13-M49 & 0.45 \\
E38-M49 & 0.40 \\
\hline
\end{tabular}

\begin{tabular}{cccccc}
\hline Tabla 1. Número y porcentaje de bandas polimórficas y monomórficas detectados en los patrones de AFLP. \\
\hline Combinación de iniciadores & Bandas monomórficas & $\%$ & Bandas polimórficas & \% & $\mathbf{N}^{\mathbf{0}}$ total de bandas \\
\hline E38-M49 & 13 & 43 & 16 & 42 & 29 \\
E13-M49 & 17 & 57 & 22 & 58 & 39 \\
Total & 30 & & 38 & 58 & 34 \\
Promedio & 15 & 44 & 19 & 56 & \\
\hline
\end{tabular}


pas nativas de Chungui. De igual forma Sánchez (2017) reportó un PIC de 0.49 en el estudio de 30 morfotipos de papas nativas en Colombia. Sin embargo, Yildirim et al. (2010), Esfahani et al. (2009) y Wang et al. (2017) lograron obtener valores altos de PIC $(0.56,0.61$ y 0.99$)$ respectivamente.

Como menciona Semagn et al. (2006), el polimorfismo no sólo depende de la combinación de iniciadores selectivos; sino también de la elección de los morfotipos y sus orígenes, siendo más polimórficos en aquellos morfotipos con orígenes diversos, debido a que en aquéllos no se da el flujo genético. Wang et al. (2017) en un estudio de 288 accesiones de papa analizados (140 accesiones del Centro Internacional de la Papa; 105 de diferentes provincias de China, 27 de otros países y 16 de fuente desconocida) reportaron un PIC de 0.99 que es muy superior a los resultados obtenidos en el presente estudio. No obstante, es necesario indicar que si bien los morfotipos muestran diversidad de formas y colores, todos los morfotipos estudiados proceden de la colección de un conservacionista de Vilcashuamán. De igual forma las colecciones que fueron estudiados por Sánchez (2017) y Gonzales y Peńa (2014) proceden de un espacio geográfico muy pequeño.

Análisis de la similitud genética.- Como se puede apreciar en la Figura 1, el valor del coeficiente de correlación cofenética " $r$ " fue de 0.7. A un coeficiente de similitud de 0.6, no se encontraron morfotipos duplicados y se determinaron 8 grupos, con similitudes genéticas que oscilaron de 0.45 a 0.81 y una media de 0.63 ; menor respecto a otros estudios, lo cual indica la alta variabilidad de los 30 morfotipos estudiados en Vilcashuamán. Sin embargo, es ligeramente inferior respecto al estudio de Sánchez (2017) quién utilizando un marcador SSR reportó una similitud media de 0.65 mostrando una mínima diferencia. Solano et al. (2007) al analizar la diversidad de papas chilenas obtuvieron una similitud media de 0.73 al igual que Wang et al. (2011) en Turquía. Del mismo modo, Esfahani et al. (2009) reportaron una similitud media para América del Norte de 0.79 y 0.77 para variedades europeas en Irán.

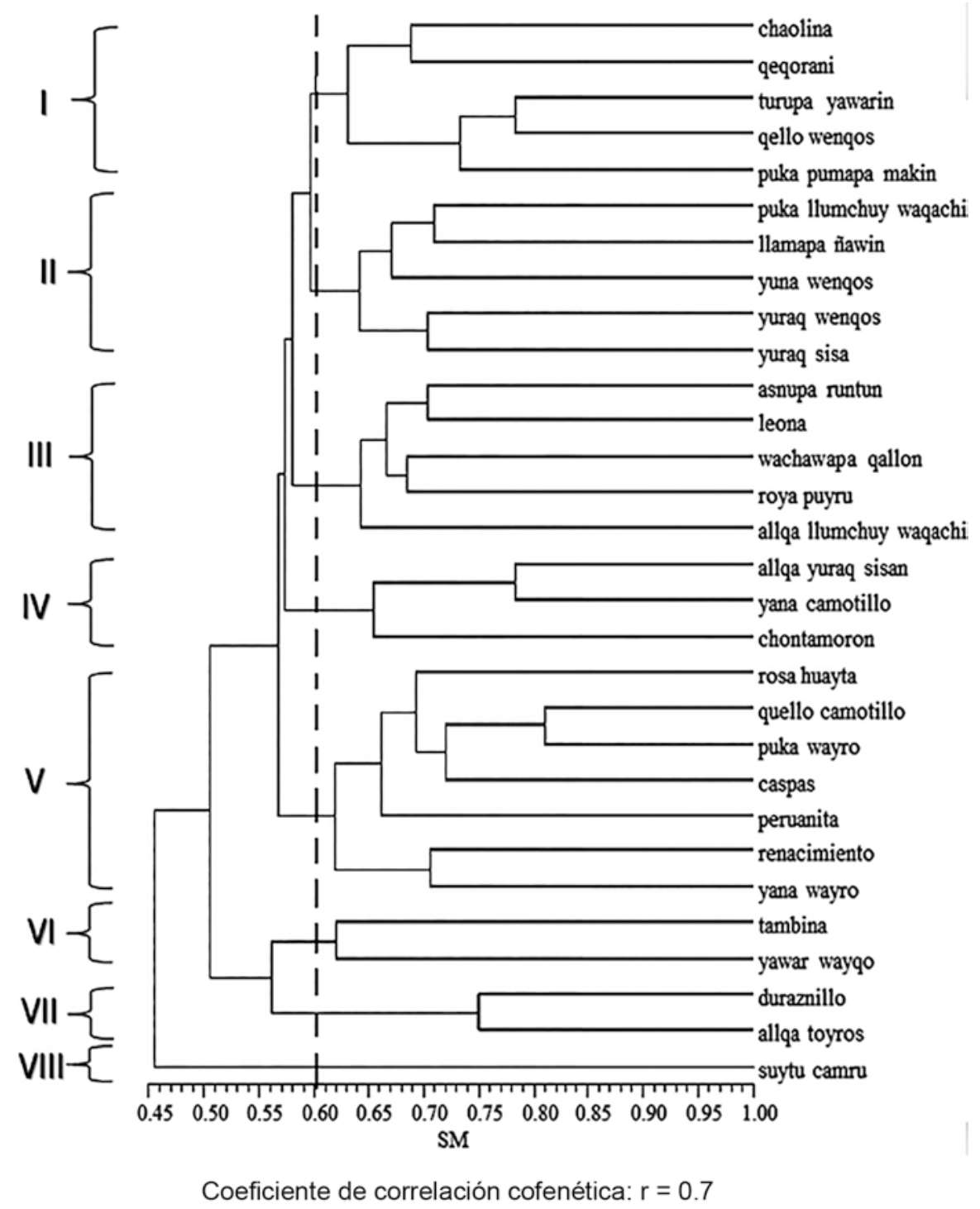

Figura 1. Dendrograma obtenido del análisis de agrupamiento con el método UPGMA de 30 morfotipos de papas nativas de la provincia de Vilcashuamán, Ayacucho, empleando el coeficiente de similitud de Simple Matching (SM). 
Respecto a la gran diversidad de papas nativas en el Perú, estudios filogenéticos han determinado su centro de origen en el altiplano peruano-boliviano (Spooner et al. 2005); sin embargo, la domesticación y gran expansión del referido tubérculo se dio durante la formación del imperio Inca y tuvo lugar en la sierra del Perú, donde se observa la mayor diversidad genética (Hawkes 1990, Sukhotu \& Hosaka 2006, Morales 2007). En efecto, Soto et al. (2013), en su estudio de diversidad genética usando microsatélites, determinaron que la región de Cusco presenta el mayor número de alelos (130 alelos), seguido de Puno (120), Ayacucho (115), Huancavelica (111) y Cajamarca (105). La poca variación probablemente se deba a que los agricultores tienden a conservar diversas variedades de papas nativas, pero entre todas ellas comparten las mismas características de utilidad para el agricultor (sabor, tamańo, productividad, etc.) que hacen que prevalezca un pool de genes "estándar".

Entre los estudios de diversidad genética realizados en el Perú, Gonzales \& Peña (2014) al estudiar la diversidad de papas nativas de Chungui, ubicado también en el departamento de Ayacucho reportaron una similitud media de 0.75, mayor respecto al obtenido en la presente investigación (0.63). Lo cual indicaría que los agricultores conservacionistas en la provincia de Vilcashuamán, mantendrían una alta diversidad genética de papas nativas y evidentemente superior al distrito de Chungui en la provincia de La Mar. Dicho resultado podría atribuirse

Tabla 4. Distribución en ocho grupos de los 30 morfotipos de papas nativas de la provincia de Vilcashuamán, Ayacucho.

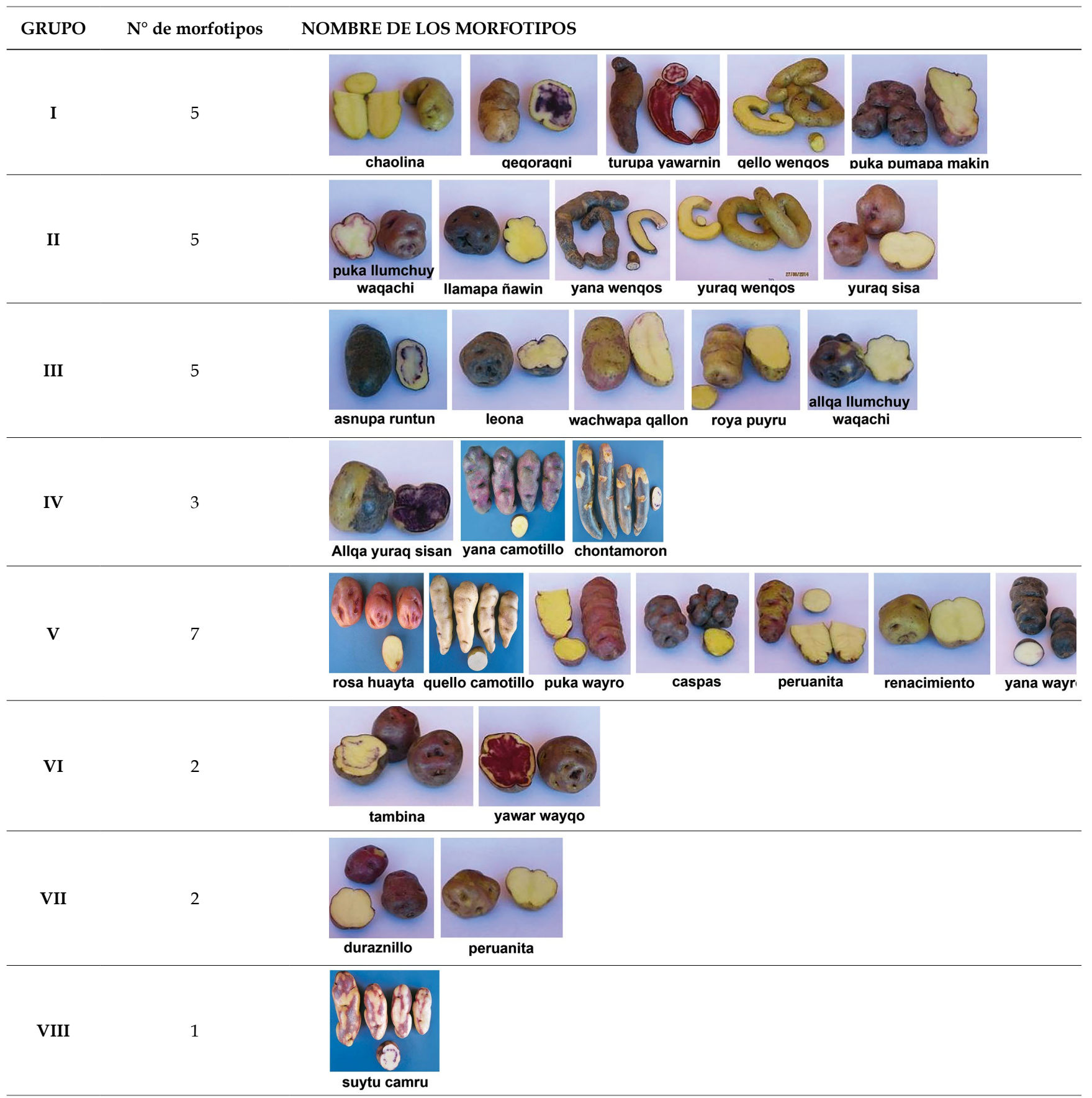


a que, en la actualidad los pobladores de esta zona que están dedicados al cultivo de las papas nativas, basan su seguridad alimentaria en el referido tubérculo y junto a su conocimiento ancestral constituyen la razón del proceso dinámico de conservación, por lo que se generan "nuevos" genotipos constantemente (Zimmerer 1991).

La premisa anterior se sustenta que entre los lugares representativos de comercio y agricultura del antiguo Perú, la ciudad de Vilcashuamán, debido a su importancia e influencia con la cultura Inca y otras según evidencias históricas y arqueológicas, fue uno de los lugares donde se realizó intercambio de cultivos como la papa (Palomino 2014, Matsumoto \& Cavero 2012, Cieza 1967), por tanto, los resultados obtenidos en presente trabajo, es la evidencia de que Vilcashuaman constituye un espacio de diversidad genética de las papas nativas, similar al Cusco como menciona Ortega (1997). No obstante, es necesario realizar otros estudios que permitan la conservación, mejoramiento genético para garantizar la seguridad alimentaria.

Análisis de agrupamiento de la diversidad genética.Aunque la mayoría de las investigaciones reportan baja correlación entre marcadores morfológicos y moleculares (Gonzales \& Peña 2014, Wang et al. 2011, Yildirim et al. 2010, Esfahani et al. 2009), en nuestro estudio, utilizando el AFLP, se obtuvo una buena correspondencia. De los ocho grupos identificados (Fig. 1 y Tabla 4), si bien todos tienen un origen común, cada grupo está determinado por morfotipos de papas nativas con caracteres similares (Tabla 3); es así, que el grupo VIII es la más distante comparado con los otros morfotipos, seguida de los grupos VII, VI, V, IV, III, II y I. Los grupos VI y VII cuyo origen es precedido por el morfotipo suytu camru, todos las muestras incluyendo el del grupo XIII, presentan una sola forma, oblonga y la piel de color rojo morado casi en su totalidad, con la presencia en algunas un color secundario de color amarillo, sobre todo alrededor de los ojos; en el grupo $\mathrm{V}$ el color de la piel empieza a tener variaciones, dos morfotipos: caspas y yana huayro, no corresponden al grupo debido a que presentan piel de color morado a negruzco y justamente ellas presentan una variación de la forma a tuberosado; pero, la pulpa en este grupo al igual que en los grupos VII y VI van de crema a amarillo en un $100 \%$ y aún persiste los ojos profundos. El grupo IV con solo tres morfotipos, presentan la forma obovada, a excepción del morfotipo allqa yuraq sisa que es comprimido y es la única que presenta la pulpa de color morado casi en su totalidad.

En el grupo III todos los morfotipos presentan forma oblonga, con pieles pigmentadas variadas de rojo, morado a negruzco, la pulpa es aún más amarilla, pero el morfotipo asnupa runtun posee en la pulpa un color secundario, formando anillos de color morado. En el grupo II, se encontró desde redondas, oblongo alargadas y tuberosados, con pieles rojizas, moradas a amarillas; pero la pulpa carece de pigmentación, todos presentan color crema. Por último, en el grupo I, dos de ellas presentan formas de fusiforme y falcado; al igual que la pulpa, de pigmentación rojo, morado, crema y amarillo; ambos grupos poseen ojos profundos, medios y sobresalientes. Los morfotipos más cercanos corresponden a qello camotillo y puka wayro del grupo V; ambas completamente diferentes; en contraste con la similitud genética de 0.81 no contrasta con la similitud fenotípica.

\section{Agradecimientos}

Nuestro agradecimiento a los conservadores de la diversidad nativa de las papas nativas, en especial al señor Julio Ochoa Gamboa, conservador y productor de las papas nativas de Vilcashuamán. Al CONCYTEC - Perú por su financiamiento parcial a través de la subvención PROCYT N 233-2008-CONCYTEC-OAJ. A los colaboradores anónimos que contribuyeron significativamente para la elaboración del presente trabajo de investigación.

\section{Literatura Citada}

Albertini E., Porceddu A., Marconi G., Barcaccia G., Pallottini L. \& Falcinelli M. (2003). Microsatellite-AFLP for genetic mapping of complex polyploids. Genome, 46(5), 824-832. DOI: http://dx.doi.org/10.1139/g03-058

Calliope S.R., Lobo M.O. \& Sammán, N.C. (2018). Biodiversity of Andean potatoes: Morphological, nutritional and functional characterization. Food chemistry, 238, 42-50. DOI: http:// dx.doi.org/10.1016/j.foodchem.2016.12.074

Canales E., O. Coto, M.T. Cornide. 2003. Variación genética e identificación de cultivares cubanos de cańa de azúcar mediante RFLP. Revista CENIC Ciencias Biológicas, 34(3):129-136.

Chambers G.K. \& MacAvoy E.S. (2000). Microsatellites: consensus and controversy. Comparative Biochemistry and Physiology Part B: Biochemistry and Molecular Biology, 126(4), 455-476. https://doi.org/10.1016/S0305-0491(00)00233-9

Cieza P. 1967. El señorío de los Incas. Instituto de Estudios Peruanos. Lima. ISBN: 980-276-394-2

CIP (Centro Internacional de la Papa). 1997. Protocolos de laboratorio de biología molecular-Tipificación genética. En: Ghislain, M., Zhang, D. \& Herrera, M. R (edit). Departamento de Recursos Genéticos. Manual de Capacitación CIP. Lima, Perú. pp. 30.

Coulibaly I., B. Revol, M. Noirot, et al. 2003. AFLP and SSR polymorphism in a Coffea interspecific backcross progeny [ $(\mathrm{C}$. heterocalyx $\times$ C. canephora) $\times$ C. canephora].Theoretical and Applied Genetics, 107(6):1148-1155. http://dx.doi. org/10.1007/s00122-003-1355-4

Dellaporta S.L., J Wood, J.B. Hicks. 1983. A plant DNA minipreparation: version II. Plant molecular biology reporter, 1(4):1921. http://dx.doi.org/10.1007/BF02712670

Esfahani S.T., B. Shiran, G. Balali. 2009. AFLP markers for the assessment of genetic diversity in European and North American potato varieties cultivated in Iran. Crop Breed. Appl. Biotechnol, 9:75-86.

Fierro F. 2004. Electroforesis de ADN. En: Cornejo A., A. Serrato, B. Rendon, M. Rocha (eds). Herramientas moleculares aplicadas en ecología: aspectos teóricos y prácticos. $1^{\text {a }}$ ed. Instituto Nacional de Ecología y Cambio Climático (INECC-SEMARNAT) (México). ISBN: 9786078246724

Gonzales J. \& G. Peña. 2014. Caracterización molecular de papas nativas (Solanum spp.) del distrito de Chungui, Ayacucho, mediante AFLP. Revista peruana de Biología, 21(3):277-282. [con acceso el 05/09/2014]. http://dx.doi.org/10.15381/ rpb.v21i3.10903

Guichoux E., Lagache L., Wagner S., Chaumeil P., Léger P., Lepais O. \& Petit R.J. (2011). Current trends in microsatellite genotyping. Molecular ecology resources, 11(4), 591-611. http://dx.doi.org/10.1111/j.1755-0998.2011.03014.x

Gutiérrez R. 2008. Papas nativas desafiando el cambio climático: Propuesta de adaptación tecnológica del cultivo de papas frente al cambio climático en Cusco y Ancash. @Soluciones Prácticas-ITDG. (Lima), p.11-30.

Hawkes J.G. 1990. The potato: evolution, biodiversity and genetic resources. Belhaven Press, London. ISBN: 1852930454

Hijmans R.J., D.M. Spooner, A.R. Salas, et al. 2002. Atlas of Wild Potatoes. Rome: International Plant Genetic Resources Institute (IPGRI), 143p. ISBN-10: 92-9043-518-6

Huamán Z. \& D.M. Spooner. 2002. Reclassification of landrace populations of cultivated potatoes (Solanum sect. Petota). American Journal of Botany, 89(6):947-965. ISSN $0256-8667$ 
Huamán Z. 1986. Botánica sistemática y morfología de la papa. $2^{\mathrm{a}}$ ed. Centro Internacional de la Papa (CIP, Lima:), 22p. (Boletín de información técnica 6).

Iglesias L.G., M. Luna, R. López. 2003. Determinación del método de extracción de ADN óptimo para el desarrollo de la técnica RAPD en megagametofitos de Pinus hartwegii Lindl. Foresta Veracruzana (México), 5(1):43-48. ISSN 1405 - 7247

Kim J.H., H. Joung, H.Y. Kim, et al. 1998. Estimation of Genetic Variation and relationship in Potato (Solanum tuberosum L.) cultivars using AFLP markers. American Journal of Potato Research, 75(2):107-112. http://dx.doi.org/10.1007/ BF02883885

Leitch L.J., M.W. Chase, M.D. Bennett. 1998. Phylogenetic analysis of DNA C-values provides evidence for a small ancestral genome size in flowering plants. Annals of Botany, (82):8594. http://dx.doi.org/10.1006/anbo.1998.0783

Madroñero I.C., Rosero J.E., Rodríguez L.E., Navia J.F. \& Benavides C.A. (2013). Morpho-agronomic characterization of promising native creole potato genotypes (Solanum tuberosum L. Andigenum group) in Nariño. Revista Temas Agrarios, 18(2), 50-66. Facultad de Ciencias Agrícolas, Universidad de Nariño, San Juan de Pasto, Colombia.

Matsumoto Y. \& Y. Cavero. 2012. Una aproximación cronológica del centro ceremonial de Campanayuq Rumi, Ayacucho. Boletín de Arqueología PUCP, (13):323-346. ISSN 1029-2004

Montalvo Fernández G., Quiroz Moreno A., Rojas Jiménez L., Quiala Mendoza E., Mederos Oroza R., Morffi Mestre H. \& Sánchez-Teyer, L. F. (2012). First report of the employment of AFLP markers in Asteraceae in Cuba. Revista Colombiana de Biotecnología, 14(2), 28-37.

Morales F.J. 2007. Sociedades precolombinas asociadas a la domesticación y cultivo de la papa (Solanum tuberosum) en Sudamérica. Revista Latinoamericana de Papa, 14(1):1-9. ISSN: 1019-6609.

Morell M.K., R. Peakall, R. Appels, et al. 1995. DNA profiling techniques for plant variety identification. Animal Production Science, 35(6), 807-819.

Moscoe L.J. \& Emshwiller E. (2015). Diversity of Oxalis tuberosa Molina: a comparison between AFLP and microsatellite markers. Genetic resources and crop evolution, 62(3), 335-347. http://dx.doi.org/10.1007/s10722-014-0154-x

Narváez R., B. Valenzuela, R. Hinrichsen. 2000. Comparación de RAPD y AFLP como métodos de identificación genética de vid basados en el estudio de fragmentos genómicos anónimos. Agricultura Técnica, 60(4):320-340. http://dx.doi. org/10.4067/S0365-28072000000400002

Nunziata A., V. Ruggieri, N. Greco, et al. 2010. Genetic diversity within wild potato species (Solanum spp.) revealed by AFLP and SCAR markers. American Journal of Plant Sciences, 1(02):95. http://dx.doi.org/10.4236/ajps.2010.12012

Ochoa C. 1999. El germoplasma de papa en Sud América: prospects for the potato in the developing world. Centro Internacional de la Papa (CIP), Lima, Perú. 1036p. ISBN 9-2906-0197-3

Ortega R. 1997. Peruvian in situ conservation of Andean crops. In: N. Maxted B. J. Ford-Lloyd. Hawkes, Chapman \& Hall (eds). Plant Genetic Conservation. (England). 307p. https://doi. org/10.1007/978-94-009-1437-7_19
Palomino Y.I.C. 2014. Evidencias arqueológicas en la avenida Calle Real, Vilcashuamán-Ayacucho. Arqueología y Sociedad, 28:43-59. ISSN: $0254-8062$

Salvatierra H. 2013. Productos nativos desafían al cambio climático. Latinoamérica en el Centro Chirapaq.

Sánchez M.A. (2017). Estudio de la variabilidad genética en accesiones de papa (Solanum tuberosum L.) mediante marcadores SSRs. Revista Ciencia y Agricultura, 14(2), 67-76.

Semagn K., Å. Bjørnstad, M.N. Ndjiondjop. 2006. Principles, requirements and prospects of genetic mapping in plants. African Journal of Biotechnology, 5(25):2569-2587.

Solano J., D. Morales, L. Anabalón. 2007. Molecular description and similarity relationships among native germplasm potatoes (Solanum tuberosum ssp. tuberosum L.) using morphological data and AFLP markers. Electronic Journal of Biotechnology, 10(3):436-443. http://dx.doi.org/10.2225/ vol10-issue3-fulltext-14

Soto J., T. Medina, Y. Aquino, et al. 2013. Diversidad genética de papas nativas (Solanum spp.) conservadas en cultivares nativos del Perú. Revista Peruana de Biología, 20(3): 215-222. http:// dx.doi.org/10.15381/rpb.v20i3.5216

Spooner D.M., K. McLean, G. Ramsay, et al. 2005. A single domestication for potato based on multilocus amplified fragment length polymorphism genotyping. Proceedings of the National Academy of Sciences of the United States of America, 102(41):14694-14699. http://dx.doi.org/10.1073/ pnas. 0507400102

Sukhotu T. \& K. Hosaka. 2006. Origin and evolution of Andigena potatoes revealed by chloroplast and nuclear DNA markers. Genome, 49:636-647. http://dx.doi.org/10.1139/G06-014

Túpac A. 2001. Postcosecha y comercialización de tubérculos andinos con énfasis en papas nativas y ulluco. En: Morales, M. \& Z. Bermúdez (eds). Perspectivas tecnológicas en el uso del germoplasma de papas nativas. Ministerio de Agricultura del Perú, Instituto Nacional de Investigación Agraria, INIA, Centro Internacional de la Papa, p.29-34. ISBN 92-90602198

Vos P., R. Hogers, M. Bleeker, et al. 1995. AFLP: a new technique for DNA fingerprinting. Nucleic acids research (England), 23(21):4407-4414. ISSN: 4407-4414

Wang F., F. Li, J. Wang, et al. 2011. Genetic diversity of the selected 64 potato germplasms revealed by AFLP markers. Molecular Plant Breeding, 2(4):22-29. http://dx.doi.org/10.5376/ mpb.2011.02.0004

Wang J., Lu H.O.U., Wang R.Y., He, M.M. \& Liu Q.C. (2017). Genetic diversity and population structure of 288 potato (Solanum tuberosum L.) germplasms revealed by SSR and AFLP markers. Journal of Integrative Agriculture, 16(11), 24342443. http://dx.doi.org/10.1016/S2095-3119(16)61619-2

Yildirim C.A.Z., M.B. Yildirim, C. Kaya, et al. 2010. Assessing genetic diversity of some potato (Solanum tuberosum L.) genotypes grown in Turkey using the AFLP marker technique. Turkish. Journal of Field Crops, 15(1): 73-78.

Zimmerer K.S. 1991. Labor shortages and crop diversity in the southern Peruvian sierra. Geographical Review, 414-432. http:// dx.doi.org/10.2307/215608 